OPEN ACCESS

Edited by:

Argel Aguilar-Valles,

Carleton University, Canada

Reviewed by:

John J. Woodward, Medical University of South Carolina,

United States

Thomas J. Papouin

Washington University Schoo of Medicine in St. Louis, United States

*Correspondence: Tak Pan Wong takpan.wong@mcgill.ca

Specialty section:

This article was submitted to Molecular Signaling and Pathways,

a section of the journal

Frontiers in Molecular Neuroscience

Received: 29 May 2021 Accepted: 16 September 2021 Published: 08 October 2021

Citation:

Tse YC, Nath M, Larosa A and Wong TP (2021) Opposing Changes

in Synaptic and Extrasynaptic

N-Methyl-D-Aspartate Receptor

Function in Response to Acute

and Chronic Restraint Stress.

Front. Mol. Neurosci. 14:716675

doi: 10.3389/fnmol.2021.716675

\section{Opposing Changes in Synaptic and Extrasynaptic N-Methyl-D-Aspartate Receptor Function in Response to Acute and Chronic Restraint Stress}

\author{
Yiu Chung Tse ${ }^{1}$, Moushumi Nath ${ }^{1,2}$, Amanda Larosa ${ }^{1,2}$ and Tak Pan Wong ${ }^{1,3 *}$ \\ ${ }^{1}$ Neuroscience Division, Douglas Research Centre, Montreal, QC, Canada, ${ }^{2}$ Integrated Program in Neuroscience, McGill \\ University, Montreal, QC, Canada, ${ }^{3}$ Department of Psychiatry, McGill University, Montreal, QC, Canada
}

A pertinent mechanism by which stress impacts learning and memory is through stress-induced plastic changes in glutamatergic transmission in the hippocampus. For instance, acute stress has been shown to alter the expression, binding, and function of the ionotropic glutamate $\mathrm{N}$-methyl-D-aspartate receptor (NMDAR). However, the consequences of chronic stress, which could lead to various stress-related brain disorders, on NMDAR function remain unclear. While most studies on NMDARs focused on these receptors in synapses (synaptic NMDARs or sNMDARs), emerging findings have revealed functional roles of NMDARs outside synapses (extrasynaptic NMDARs or exNMDARs) that are distinct from those of sNMDARs. Using a restraint stress paradigm in adult rats, the objective of the current study is to examine whether sNMDARs and exNMDARs in the hippocampus are differentially regulated by acute and chronic stress. We examined sNMDAR and exNMDAR function in dorsal CA1 hippocampal neurons from brain slices of adult rats that were acutely ( 1 episode) or chronically (21 daily episodes) stressed by restraint (30 min). We found that acute stress increases sNMDAR but suppresses exNMDAR function. Surprisingly, we only observed a reduction in exNMDAR function after chronic stress. Taken together, our findings suggest that sNMDARs and exNMDARs may be differentially regulated by acute and chronic stress. Most importantly, the observed suppression in exNMDAR function by both acute and chronic stress implies crucial but overlooked roles of hippocampal exNMDARs in stress-related disorders.

Keywords: brain slice, corticosterone, electrophysiology, hippocampus, synaptic plasticity

\section{INTRODUCTION}

$\mathrm{N}$-methyl-D-aspartate receptor (NMDAR) is an ionotropic glutamate receptor that mediates synaptic plasticity and neuronal fate (Hardingham and Bading, 2003; Lau and Zukin, 2007; Paoletti et al., 2013). NMDARs also play crucial roles in mediating the biological impacts of stress on the nervous system. For instance, the facilitating effect of acute stress on pain sensation (Alexander et al., 2009) and fear conditioning (Shors and Servatius, 1995; Shors and Mathew, 1998) can be 
abolished by NMDAR antagonists. The atrophy and loss of spines on cortical and hippocampal neurons in stressed rodents can also be rescued by the blockade or the genetic knockdown of NMDARs (Magarinos and McEwen, 1995; Christian et al., 2011; Li N. et al., 2011; Martin and Wellman, 2011). The impact of stress on NMDAR expression and function has been extensively examined. Acute stress can increase the expression (Gore et al., 2000; Yamamoto et al., 2008; Yuen et al., 2012), binding (Krugers et al., 1993) and function of NMDARs (Yuen et al., 2009, 2011). The effect of chronic stress on NMDAR expression and function is less clear. Previous studies have revealed increased (Calabrese et al., 2012; Costa-Nunes et al., 2014; Pacheco et al., 2017), decreased (Pacheco et al., 2017) or stable (Schwendt and Jezova, 2000; Nasca et al., 2015) NMDAR expression in the hippocampus after chronic stress exposure. These somewhat conflicting findings may be due to differences in the type and duration of stressors used in these studies. Finally, only a few studies have examined the impact of chronic stress on NMDAR function (Karst and Joels, 2003; Yuen et al., 2012; Tse et al., 2019). Since chronic stress is associated with the pathogenesis of mood disorders (McEwen, 2003; Hammen, 2005), it is imperative to further examine if NMDAR function can also be regulated by chronic stress.

While our current understanding of the functional roles of NMDARs is based on studies of these receptors in synapses (sNMDARs), emerging findings have revealed functional roles for NMDARs that are located outside synapses (extrasynaptic NMDARs or exNMDARs). With high levels in the early postnatal age (Tovar and Westbrook, 1999), the proportion of exNMDAR decreases with development. However, close to $30 \%$ NMDARs in adult hippocampal slices remain extrasynaptic (Papouin et al., 2012). In addition to its substantial presence in the brain, exNMDARs can be physiologically activated through various means (for review, see Petralia et al., 2010; Papouin and Oliet, 2014). For instance, exNMDARs can be activated by glutamate from extracellular space (Le Meur et al., 2007), astrocytes (Carmignoto and Fellin, 2006; Nie et al., 2010) and repetitive stimulation of glutamate synapses (Suzuki et al., 2008). Back-propagating actional potentials also facilitate exNMDAR activation (Wu et al., 2012). Functional roles mediated by exNMDARs include neuronal synchrony (Fellin et al., 2004), synaptic computation (Oikonomou et al., 2012) and plasticity (Lu et al., 2001; Liu et al., 2013). These findings provide a more complete picture of exNMDAR physiology in addition to its contribution to neuronal death (Hardingham and Bading, 2010; Parsons and Raymond, 2014). Notably, these emerging functional roles of exNMDARs beg the question of whether these receptors are subjected to stressrelated regulation.

Using a restraint stressor, the objective of this study is to examine the impact of acute and chronic stress on SNMDAR and exNMDAR functions in the hippocampal CA1 region, a region that is sensitive to stress and highly implicated in stressrelated mood disorders (Brown et al., 1999; McEwen, 1999; Campbell and Macqueen, 2004; Duman and Monteggia, 2006). We found that exNMDAR function can be reduced by both acute and chronic stress.

\section{MATERIALS AND METHODS}

\section{Animals}

All care and use of animals were in accordance with the guidelines and policies of the Canadian Council on Animal Care and approved by the Facility Animal Care Committee of the Douglas Research Centre (DRC), McGill University (animal use protocol number 2010-5935). Adult (8-12-week old) male Sprague Dawley (SD) rats were obtained from Charles River Laboratories. They were housed in the DRC animal facility and maintained on a 12/12 light cycle with lights on at 08:00 AM. Food and water were available ad libitum. Rats were used for experiments after being housed in the animal facility for at least 1 week to reduce the influence of stress from transportation.

\section{Stress Paradigm}

Each episode of restraint stress was performed by putting a rat into a DecapiCone (BrainTree Scientific) for $30 \mathrm{~min}$. For acute stress, rats were sacrificed $30 \mathrm{~min}$ after the end of one episode of restraint stress. For chronic stress, rats were restrained once daily for 21 days. Rats were sacrificed within a week after chronic stress (3.0 \pm 0.4 days) for electrophysiological recording. Control rats for the acute stress and chronic stress experiments were handled daily for 1 and 21 days, respectively.

\section{Brain Slice Preparation}

Unless specified otherwise, all materials and chemicals were purchased from Sigma Aldrich. Rats were anesthetized with isoflurane (5\%) and decapitated using a guillotine. Trunk blood was collected after decapitation in EDTA-containing tubes to prepare serum for CORT (corticosterone) ELISA (Abcam. ELISA was performed using manufacturer's procedures). Coronal brain slices were cut in a hyperosmotic, ice-cold and carbogenated (5\% $\mathrm{CO}_{2}, 95 \% \mathrm{O}_{2}$ ) slice-cutting solution (in mM: 252 sucrose, 2.5 $\mathrm{KCl}, 4 \mathrm{MgCl}_{2}, 0.1 \mathrm{CaCl}_{2}, 1.25 \mathrm{KH}_{2} \mathrm{PO}_{4}, 26 \mathrm{NaHCO}_{3}$ and 10 glucose, $\sim 360 \mathrm{mOsmol} / \mathrm{L}$ ) using a vibrating blade microtome (Leica). Slices were then transferred to carbogenated artificial cerebrospinal fluid (aCSF; in mM: $125 \mathrm{NaCl}, 2.5 \mathrm{KCl}, 1 \mathrm{MgCl}_{2}$, $2 \mathrm{CaCl}_{2}, 1.25 \mathrm{NaH}_{2} \mathrm{PO}_{4}, 26 \mathrm{NaHCO}_{3}$ and 25 glucose, 2310 $\mathrm{mOsmol} / \mathrm{L}$ ) at $32^{\circ} \mathrm{C}$ for $1 \mathrm{~h}$, followed by room temperature incubation before recordings.

\section{Electrophysiology}

\section{General Procedures}

All recordings were performed in the dorsal hippocampal CA1 region at room temperature. Synaptic responses were evoked by stimulating the Schaffer collateral-commissural pathway through a tungsten bipolar electrode $(F H C)$ using constant current pulses $(0.08 \mathrm{~ms})$ at $0.05 \mathrm{~Hz}$. Electrophysiological data were amplified by the Multiclamp 700 B amplifier (Molecular Devices), digitized by the Digidata 1440 (Molecular Devices), and stored in a PC for offline analysis.

\section{Field Excitatory Postsynaptic Potential}

Recordings of evoked AMPA receptor (AMPAR)-mediated field excitatory postsynaptic potentials (fEPSPs) were performed with 
an aCSF-containing glass pipette in the stratum radiatum. $\mathrm{GABA}_{\mathrm{A}}$ receptors $\left(\mathrm{GABA}_{\mathrm{A}} \mathrm{Rs}\right)$ were blocked by bicuculline methobromide $(10 \mu \mathrm{M})$ and picrotoxin $(20 \mu \mathrm{M})$. To record NMDAR-mediated-fEPSP, we reduced the concentration of $\mathrm{MgCl}_{2}$ in aCSF to $0.05 \mathrm{mM}$. AMPARs were blocked by DNQX $(20 \mu \mathrm{M})$.

\section{Excitatory Postsynaptic Current}

Recordings of evoked excitatory postsynaptic currents (EPSCs) from CA1 pyramidal neurons were performed using a patch pipette containing (in $\mathrm{mM}$ ) 110 Cs-gluconate, $17.5 \mathrm{CsCl}, 2$ $\mathrm{MgCl}_{2}, 0.5$ EGTA, 10 HEPES (Wisent Inc.), 4 ATP, and 5 QX314 (Alomone Labs), with the $\mathrm{pH}$ adjusted to 7.2 by $\mathrm{CsOH}(\sim 290$ $\mathrm{mOsmol} / \mathrm{L})$. Only recordings with an access resistance lower than $20 \mathrm{M} \Omega$ were kept. We did not observe differences between the input resistance of CA1 neurons from different animal groups (control: $152.3 \pm 6.4 \mathrm{M} \Omega$; acute stress: $159.9 \pm 7.0 \mathrm{M} \Omega$; chronic stress: $162.1 \pm 14.0 \mathrm{M} \Omega$ ). The AMPAR- and NMDAR-mediated component of EPSCs were evoked while voltage clamping the membrane potential at -60 and $+40 \mathrm{mV}$, respectively. In EPSCs evoked at $+40 \mathrm{mV}$, the magnitude of EPSC at $150 \mathrm{~ms}$ poststimulation was used to represent the NMDAR component, since the AMPAR component has returned to baseline at this time point (see Figure 1A). To examine exNMDAR currents, EPSCs mediated by sNMDARs were recorded while voltage clamping the neuron at $+40 \mathrm{mV}$ in the presence of DNQX to block the AMPAR component and maintained at around $100 \mathrm{pA}$. After a $>90 \%$ blockade of sNMDAR currents by a 30-min-long application of MK801 (40 $\mu \mathrm{M}$, EPSCs were evoked at $0.1 \mathrm{~Hz}$ during MK801 blockade), exNMDAR currents were induced by (i) the blockade of glutamate transporters by DL-threo$\beta$-benzyloxyaspartic acid (TBOA, $20 \mu \mathrm{M}$; Milnerwood et al., 2010; Li S. et al., 2011), or (ii) a brief 10-pulse tetanus at $100 \mathrm{~Hz}$ as previously described (Luscher et al., 1998).

\section{Statistical Analysis}

All data are presented as mean \pm SEM. Statistical analyses were performed using JMP 13. Normality of data was examined using the Shapiro-Wilk test. Student's $t$-tests were used for comparisons of normally distributed data between two groups. Two-way ANOVA was also used to compare the effect of stress on the input/output relationship of evoked fEPSPs. For data that are not normally distributed, Mann-Whitney tests were used for comparisons between two groups and Kruskal-Wallis tests and post hoc Wilcoxon tests were used for comparing data from three groups. Statistical significance (2-tailed) was set at $p<0.05$.

\section{RESULTS}

\section{Acute Stress Enhances sNMDAR and Suppresses exNMDAR Functions}

We first examined the effect of acute restraint stress on SNMDAR and exNMDAR functions in the hippocampal CA1 region of control and acutely stressed adult male rats. Acute restraint stress significantly increased CORT blood levels [CORT levels in controls: $48.5 \pm 16.8 \mathrm{ng} / \mathrm{ml}$; CORT levels in stressed rats:

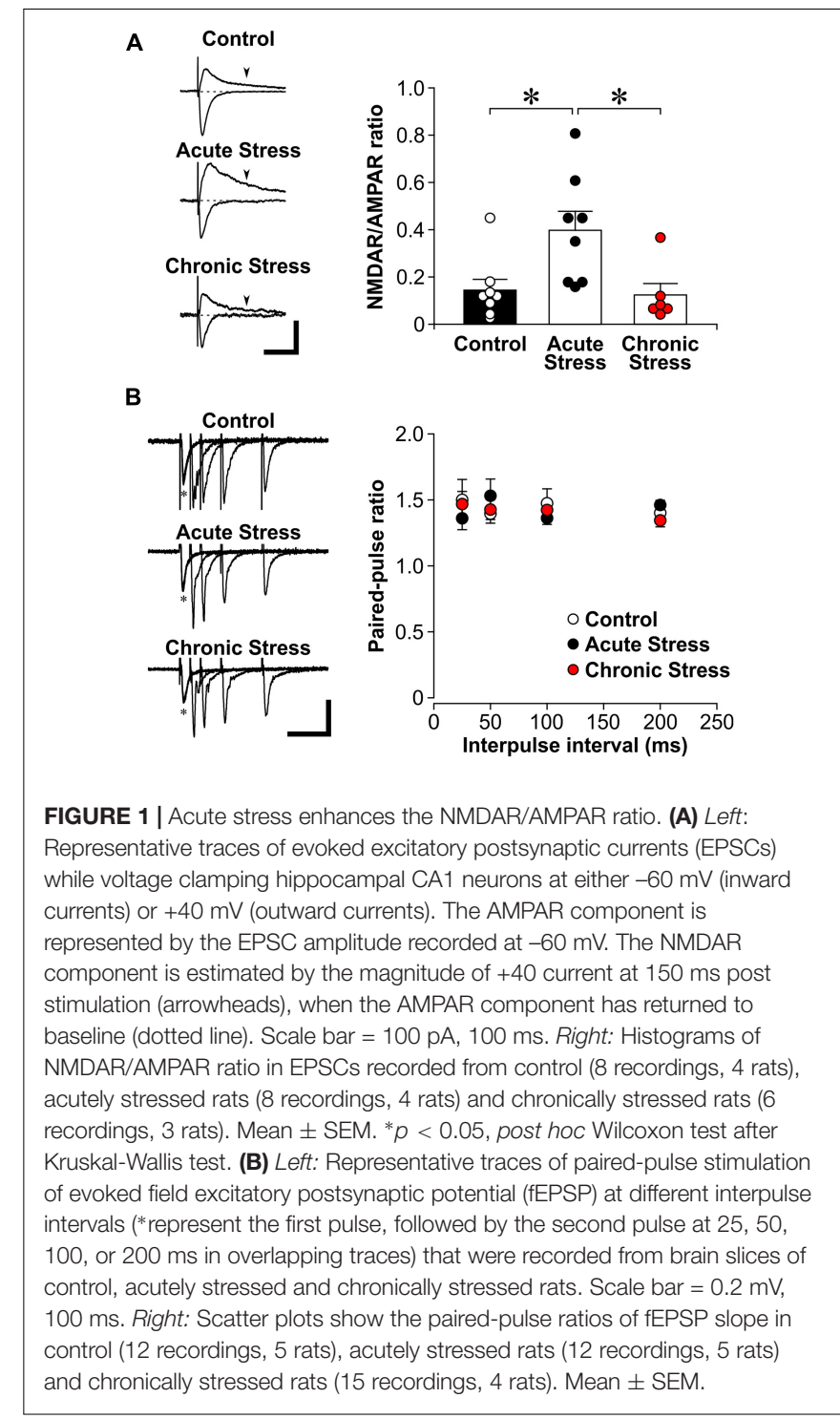

$495.4 \pm 36.8 \mathrm{ng} / \mathrm{ml} ; t(4)=13.5 ; p=1.73 \mathrm{E}-04]$. In the presence of AMPAR and GABA $\mathrm{R}$ antagonists, we isolated evoked fEPSPs that were mediated by sNMDARs (Figure 2A). To compare fEPSP between slices, we examined the relationship between the amplitude of fiber volley ( $f v$; input), which represents the depolarization of synaptic inputs, and the slope of fEPSP (output). We found that acute stress significantly increases the input/output relationship of NMDAR-mediated fEPSPs when compared to controls \{two-way ANOVA, stress- $[F(1$, $10)=5.16, p=0.046] ; f v$ amplitudes (as repeated measures) $[F(4,40)=142.5, p=4.24 \mathrm{E}-23]$; stress $\times f v$ amplitudes$[F(4,40)=3.46, p=0.016]\}$. However, acute stress did not affect the input/output relationship of AMPAR-mediated fEPSPs when compared to controls \{Figure 2B; two-way ANOVA, stress- $[F(1,23)=0.01, p=0.918] ; f v$ amplitudes (as repeated measures $)-[F(4,92)=55.2, p=1.21 \mathrm{E}-23]$; stress $\times f v$ amplitudes- $[F(4,92)=0.58, p=0.681]\}$. 
A

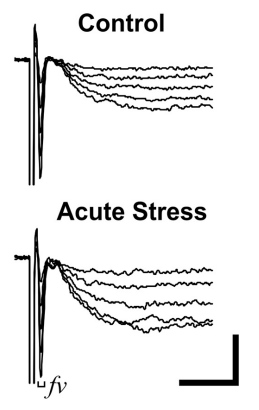

B
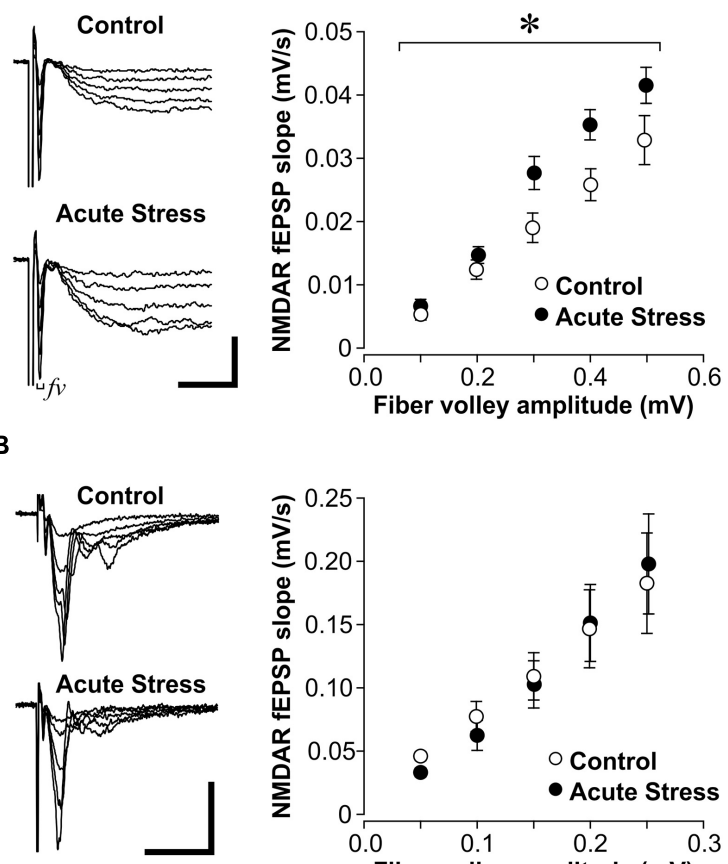

Fiber volley amplitude $(\mathrm{mV})$

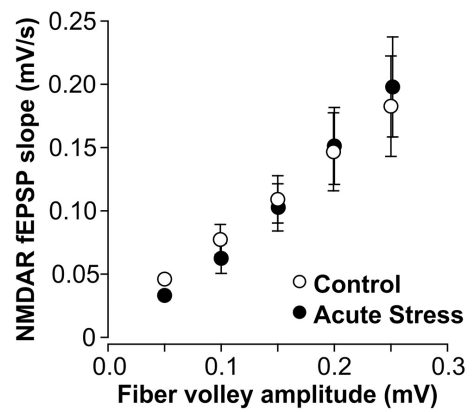

C

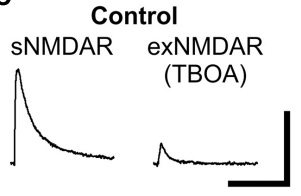

Acute Stress SNMDAR EXNMDAR
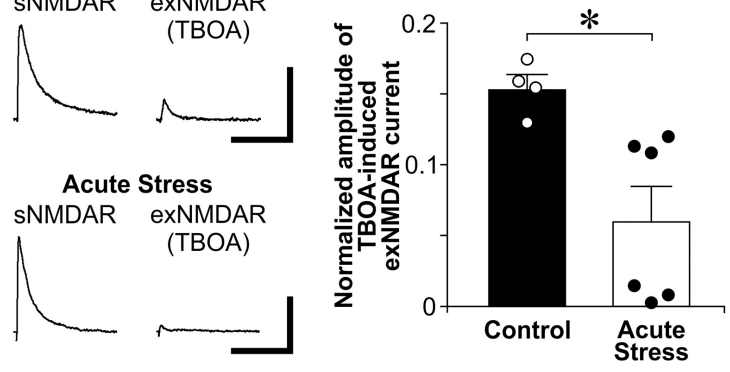

D
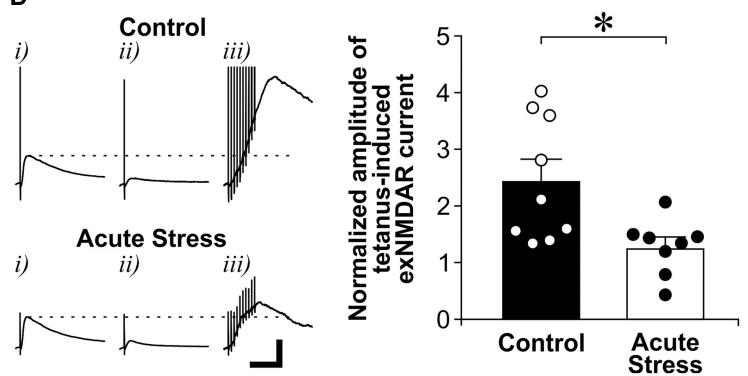

FIGURE 2 | Acute stress enhances sNMDAR and reduces exNMDAR functions. (A) Left: Representative traces of evoked NMDAR-mediated evoked field excitatory postsynaptic potential (fEPSP) that were recorded from brain slices of control and acutely stressed rats (restrained for $30 \mathrm{~min}$ ). Scale bar $=0.2 \mathrm{mV}, 10 \mathrm{~ms}$. Right: Scatter plots show the relationship between fiber volley $(f v)$ amplitude and the slope of NMDAR fEPSP in control (6 recordings, 3 rats) and stressed rats (6 recordings, 3 rats). Mean \pm SEM. ${ }^{*} p<0.05$, interaction between the effect of stress and fiber volley amplitudes [repeated measures ANOVA, $F(4,40)=3.46]$. (B) Left: Representative traces of evoked AMPAR-mediated fEPSP that were recorded from brain slices of control and

(Continued)
FIGURE 2 | (Continued)

acutely stressed rats. Scale bar $=0.4 \mathrm{mV}, 25 \mathrm{~ms}$. Right: Scatter plots show the relationship between fiber volley amplitude and the slope of AMPAR fEPSP in control (15 recordings, 4 rats) and stressed rats (10 recordings, 3 rats). Mean \pm SEM. (C) Left: Representative traces of SNMDAR and TBOA-induced exNMDAR currents that were recorded from brain slices of control and acutely stressed rats. Scale bar $=50$ pA, 500 ms. Right: Histogram shows the normalized amplitude of exNMDAR currents recorded from slices of control (4 recordings, 4 rats) and acutely stressed rats (6 recordings, 3 rats). Mean \pm SEM. * $p<0.05$, Student's $t$-test. (D) Left: Representative traces of evoked NMDAR mediated currents that were recorded from brain slices of control and acutely stressed rats before (i) and after a 30 min-long MK801 blockade (ii). Five minutes after washing out MK801, exNMDAR currents were induced by a brief tetanus stimulation [iii, 10-pulse $(100 \mathrm{~Hz})]$. Dotted lines represent the level of sNMDAR current before MK801 blockade. Scale bar $=100$ pA, 100 ms. Right: Histograms of normalized amplitude of exNMDAR currents recorded from brain slices of control (9 recordings, 9 rats) or acutely stressed rats (8 recordings, 5 rats). Mean \pm SEM. ${ }^{*} p<0.05$, Student's $t$-test.

We next examined the impact of acute stress on exNMDAR function (Figure 2C). To isolate exNMDAR-mediated currents, we first blocked evoked sNMDAR currents by MK801. MK801 produced a sustained use-dependent blockade of NMDARs (Tse et al., 2019). We next induced a spillover of synaptic glutamate release by blocking glutamate transporter with TBOA to activate exNMDARs (Milnerwood et al., 2010; Li S. et al., 2011). Compared to controls, we found that acute stress reduces the normalized amplitude of exNMDAR currents [normalized by the amplitude of sNMDAR currents before MK801 blockade from the same cell; $t(8)=3.06, p=0.016]$. Apart from using TBOA, we used a brief tetanus (Luscher et al., 1998; 10-pulse at $100 \mathrm{~Hz}$ ) to increase extracellular glutamate levels after MK801 blockade of sNMDAR to activate exNMDARs in another set of slices from stressed and control rats. Like the TBOA findings, we found that the amplitude of exNMDAR current in slices from acutely stressed rats is significantly smaller than that in slices from control rats (Figure 2D; $\mathrm{U}=11, p=0.018$ ). These findings suggest that acute stress enhances SNMDAR and reduces exNMDAR function in dorsal hippocampal CA1 neurons.

\section{Acute Stress Reduces Ambient Glutamate-Mediated exNMDAR Currents}

Since exNMDAR currents induced by TBOA and a brief tetanus after MK801 blockade was normalized by sNMDAR currents, the reduced exNMDAR function may also be due to the enhanced sNMDAR function caused by stress. To address this limitation, we used another method to directly measure exNMDAR function. exNMDARs can be activated by ambient levels of glutamate in brain slices, which can be revealed by the shift in whole-cell holding currents caused by NMDAR antagonists such as MK801 or APV (Le Meur et al., 2007; Papouin et al., 2012). To show that the shift in whole-cell currents caused by a NMDAR antagonist does not affect sNMDAR function, we recorded sNMDAR-mediated EPSCs from CA1 pyramidal neurons before and after perfusing slices with MK801. Although MK801 treatment caused a shift in whole-cell currents, it did not affect the amplitude of sNMDAR EPSCs (Figures 3A,B). Next, we 
A

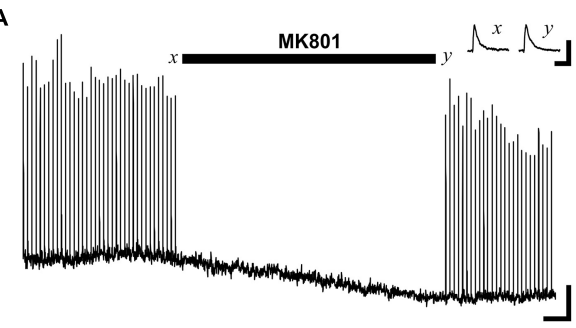

B

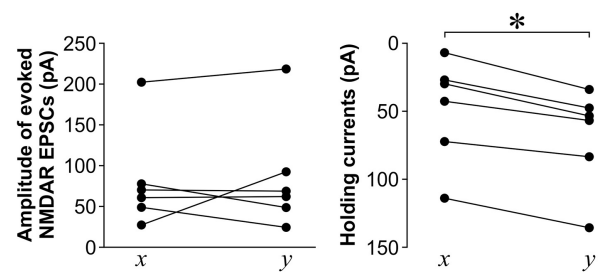

C
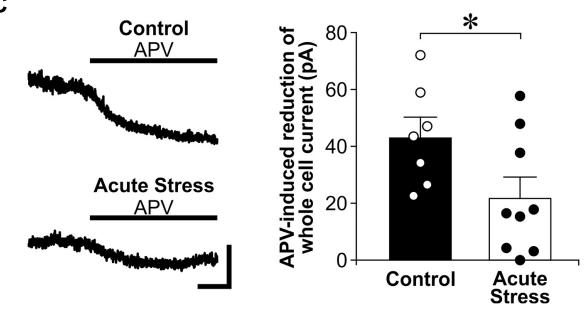

FIGURE 3 | Acute stress reduces exNMDAR currents mediated by ambient glutamate. (A) Representative trace shows the effect of MK801 on evoked NMDAR-mediated EPSCs and whole-cell holding currents recorded from a CA1 neuron. Stimulations for evoking EPSCs were stopped at $x$ and during MK801 application. MK801 reduced whole-cell holding currents. However, the amplitudes of evoked NMDAR-mediated EPSCs were not affected when stimulation was resumed at time $y$. The reduction of EPSC amplitude near the end of the trace was likely due to residual levels of MK801 in the recording chamber. Scale bar $=40$ pA, 1 min. Inset: traces of sNMDAR before $(x)$ and after (y) MK801 application. Scale bar $=200$ pA, 200 ms. (B) Line plots show changes in evoked NMDAR-mediated EPSCs (left; 6 recordings, 3 rats) and whole-cell holding currents (right; 6 recordings, 3 rats) before $(x)$ and after MK801 application (y). ${ }^{*} p<0.05$, paired Student's $t$-test; current at time $x$ vs. time y. (C) Left: Representative traces of APV $(50 \mu \mathrm{M})$-induced blockade of whole-cell current of CA1 neurons in brain slices from control or acutely stressed rats. Scale bar $=40 \mathrm{pA}, 1 \mathrm{~min}$. Right: Histograms of the APV-induced blockade of whole-cell currents recorded from hippocampal slices of control (7 recordings, 3 rats) or acutely stressed rats (9 recordings, 4 rats). Mean \pm SEM. ${ }^{*} p<0.05$, Student's $t$-test.

compared the shift in whole-cell currents caused by APV between control and acutely stressed rats. Compared to slices from control rats, we found that APV-sensitive holding currents recorded from CA1 pyramidal neurons are significantly smaller in slices from acutely stressed rats [Figure 3C; $t(14)=2.17, p=0.048$ ]. These findings corroborated data from the spillover experiments to show that acute stress reduces exNMDAR function.

\section{Chronic Stress Suppresses exNMDAR Functions}

After being stressed by restraint daily for 21 days, chronically stressed rats displayed lower body weight gain over 21 days of stress than control rats [Figure $4 \mathrm{~A} ; t(13)=3.65, p=0.003$ ]. In addition, we found higher plasma CORT levels in chronically stressed rats when compared to non-stressed controls rats $[t(13)=2.74, p=0.017]$. Surprisingly, we found no difference in sNMDAR function between chronically stressed and control rats $\{$ Figure 4B; two-way ANOVA, stress- $[F(1,13)=0.291$, $p=0.599] ; f v$ amplitudes (as repeated measures) $-[F(4$, $52)=125.0, p=5.24 \mathrm{E}-26]$; stress $\times f v$ amplitudes $-[F(4$, $52)=0.546, p=0.703]\}$. Chronic stress also did not affect the input/output function of AMPAR-mediated fEPSP \{Figure 4C; two-way ANOVA, stress- $[F(1,21)=0.113, p=0.741] ; f v$ amplitudes (as repeated measures) $-[F(4,84)=32.2, p=2.94 \mathrm{E}$ 17]; stress $\times f v$ amplitudes $-[F(4,84)=0.733, p=0.572]\}$. Nonetheless, chronically stressed rats displayed lower TBOAinduced exNMDAR currents than control rats \{Figure 4D, $[t(10)=2.69, p=0.023]\}$. In addition, chronically stressed rats also showed lower APV-induced shifts in holding currents of CA1 neurons than control rats $\{$ Figure $4 \mathbf{E},[t(8)=3.31, p=0.011]\}$. Taken together, chronic stress reduced exNMDAR function.

To further show the specific impact of acute stress on sNMDAR function, we performed whole-cell recordings of the NMDAR/AMPAR ratio of evoked EPSC. We found that acute stress significantly increased the NMDAR/AMPAR ratio of evoked EPSC when compared to control and chronic stress (Figure 1A; Kruskal-Wallis test, $Z=9.75, p=0.008$; post hoc Wilcoxon tests: control vs. acute stress: $p=0.010$; acute stress vs. chronic stress: $p=0.012$; control vs. chronic stress: $p=0.478$ ).

\section{Acute and Chronic Stress Do Not Affect Presynaptic Glutamate Release}

High presynaptic glutamate release might recruit exNMDARs during MK801 blockade and result in the reduced exNMDAR currents we observed in slices from stressed rats. If acute or chronic stress enhances presynaptic glutamate release, we expect MK801 would need a shorter time to block sNMDAR currents. However, comparing the time for blocking $50 \%$ of sNMDAR currents by MK801 between slices from control and acutely stressed rats (control: $10.2 \pm 1.4 \mathrm{~min}$; acute stress: $9.2 \pm 1.0 \mathrm{~min} ;[t(10)=0.58 ; p=0.578])$, or between control and chronically stressed rats (control: $9.8 \pm 0.7 \mathrm{~min}$; chronic stress: $9.0 \pm 1.1 \mathrm{~min} ;[t(10)=0.60 ; p=0.562]$ ), revealed no differences. We also compared the paired-pulse ratios of fEPSP at different interpulse intervals (25-200 ms) in slices from control and stressed rats. We found no differences in paired-pulse facilitation between the 3 groups \{Figure 1B; two-way ANOVA, animal groups $-[F(2,34)=0.370, p=0.694]$; interpulse interval (as repeated measures) $-[F(3,102)=1.6, p=0.187]$; animal groups $\times$ interpulse interval $-[F(6,102)=0.814, p=0.561]\}$. Together, these findings suggest that the impact of stress on exNMDAR function is not related to changes in presynaptic glutamate release.

\section{DISCUSSION}

Stress-induced plasticity of NMDAR function depends on stress duration and the locations of these receptors. We show in this study that acute stress enhanced sNMDAR and reduced 


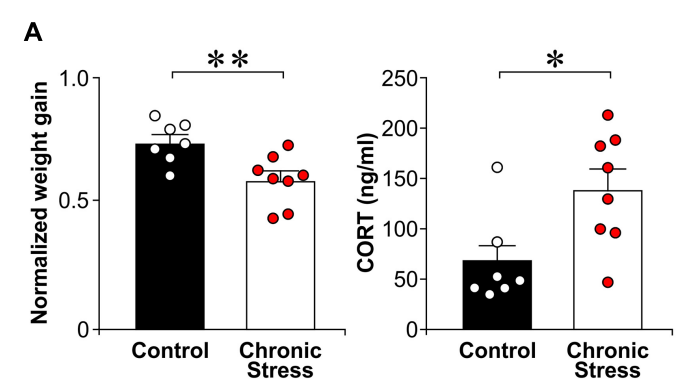

.

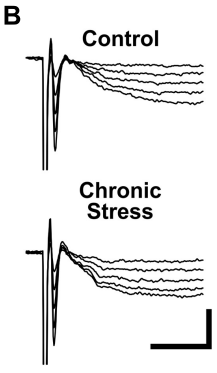

C
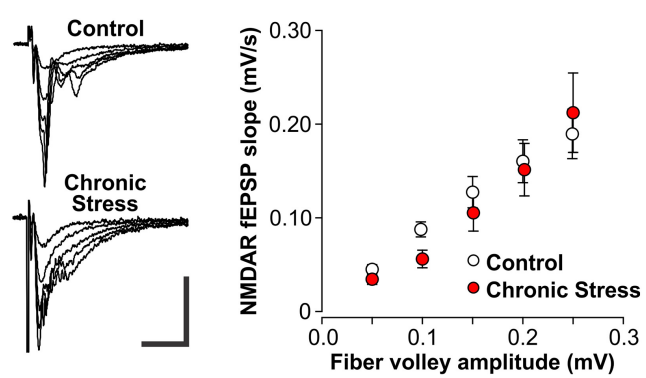

D
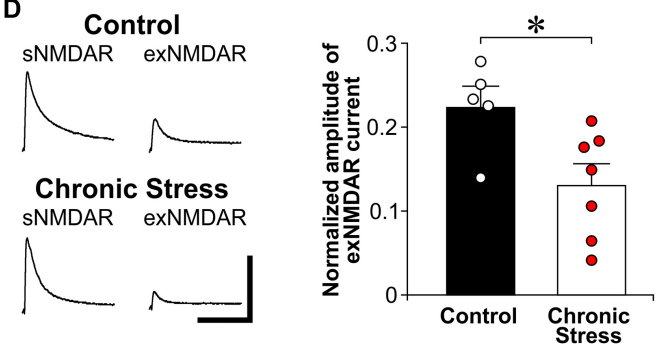

E
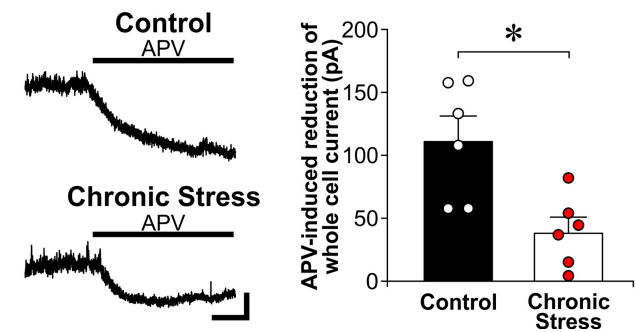

FIGURE 4 | Chronic stress reduces exNMDAR functions. (A) Left: Histograms of normalized weight gain of control $(n=7)$ and chronically stressed rats ( $n=8)$ in 21 days. Right: Histograms of CORT levels in trunk blood in control $(n=7)$ and chronically stressed rats $(n=8)$ collected right before brain slice preparation. Stressed rats were sacrificed 7 days within the end of chronic

(Continued)
FIGURE 4 | (Continued)

restraint stress. Mean \pm SEM. ${ }^{*} p<0.05,{ }^{* *} p<0.01$, Student's $t$-test.

(B) Left: Representative traces of evoked NMDAR-mediated field excitatory postsynaptic potential (fEPSP) that were recorded from brain slices of control and chronically stressed rats. Scale bar $=0.2 \mathrm{mV}, 40 \mathrm{~ms}$. Right: Scatter plots show the relationship between fiber volley amplitude and the slope of NMDAR fEPSP in control (7 recordings, 4 rats) and stressed rats (8 recordings, 4 rats). Mean \pm SEM. (C) Left: Representative traces of evoked AMPAR-mediated fEPSP that were recorded from brain slices of control and chronically stressed rats. Scale bar $=0.4 \mathrm{mV}, 25 \mathrm{~ms}$. Right: Scatter plots show the relationship between fiber volley amplitude and the slope of AMPAR fEPSP in control (10 recordings, 4 rats) and stressed rats (13 recordings, 4 rats). Mean \pm SEM. (D) Left: Representative traces of sNMDAR and TBOA-induced exNMDAR currents from brain slices of control or chronically stressed rats. Scale bar $=50$ pA, 500 ms. Right: Histogram shows the normalized amplitude of exNMDAR currents recorded from brain slices of control (5 recordings, 4 rats) and chronically stressed rats (7 recordings, 6 rats). Mean \pm SEM. ${ }^{*} p<0.05$, Student's $t$-test. (E) Left: Representative traces of APV $(50 \mu \mathrm{M})$-induced blockade of whole-cell current of CA1 neurons in brain slices from control and chronically stressed mice. Scale bar $=40$ pA, 1 min. Right: Histograms of the APV-induced blockade of whole-cell currents recorded from control (6 recordings, 3 rats) and chronically stressed mice (6 recordings, 3 rats). Mean \pm SEM. ${ }^{*} p<0.05$, Student's $t$-test.

exNMDAR functions. Interestingly, reduced exNMDAR function was also induced by chronic stress. Apart from sNMDARs (Yuen et al., 2009; Tse et al., 2011), we reveal in this study that exNMDARs are highly susceptible to stress-induced regulation.

Our findings are in parallel to previous findings that while acute stress exposure increases sNMDAR function, this increase may be transient and may not be sustained by repeated exposure to the same stressor. In the prefrontal cortex (PFC), both acute restraint and forced swim stress have been shown to increase postsynaptic AMPAR and NMDAR responses (Yuen et al., 2009, 2012). However, recording these postsynaptic responses from rats that were stressed by a similar restraint stressor daily revealed a decrease in synaptic AMPAR and NMDAR function after 7 days when compared to controls (Yuen et al., 2012). In the same study, 7-day restraint stress did not affect AMPAR function in the CA1. Finally, both synaptic AMPAR and NMDAR responses recorded from granule cells of the dentate gyrus were not affected in rats that have received 21 daily episodes of chronic unpredictable stress (Karst and Joels, 2003). Since there was a mean delay of 3 days between the end of chronic stress and electrophysiology recording, we cannot rule out the possibility that sNMDAR was enhanced immediately after chronic stress but returned to baseline when we sacrificed the animals. Even if sNMDAR function is transiently increased after chronic stress, this change is unlike the persistent decrease in exNMDAR function.

We found that exNMDAR function was reduced by both acute and chronic restraint stress. Although glutamate spillover caused by either a brief tetanus or the blockade of glutamate transporters has been used to estimate exNMDAR function, these indirect methods have limitations. To compare exNMDAR function between slices, exNMDAR function was normalized by sNMDAR currents from the same slice in the current study. The increase in SNMDAR function caused by acute stress could be a factor that may lead to a decrease in normalized exNMDAR function. The more direct method of estimating 
exNMDAR function by using the blockade of exNMDARmediated whole-cell currents was used to address this limitation (Le Meur et al., 2007; Yao et al., 2018). The spillover method may also target a specific population of exNMDARs. In Figure 3, we found that the MK801 treatment we used to block sNMDARs could block exNMDARs that are activated by ambient glutamate. This finding suggests that the spillover approach recruits a different population of exNMDARs (also called perisynaptic NMDARs) (Mitani and Komiyama, 2018) that are not sensitive to ambient glutamate. Despite these limitations, we found that both acute and chronic stress reduce exNMDAR currents that are sensitive to ambient glutamate. In addition, chronic stress reduced TBOA- and tetanus-induced exNMDAR currents without affecting sNMDAR function. Together, our findings suggest that exNMDARs are reduced by both acute and chronic stress.

Mechanisms underlying the reduction of exNMDAR function after stress remains to be determined. After acute stress, the reduction of exNMDARs may be due to surface trafficking of these receptors to synapses for enhancing sNMDAR function (e.g., see Tovar and Westbrook, 2002; Groc et al., 2006). Nonetheless, how a reduction of exNMDARs was maintained when sNMDAR function is no longer enhanced after chronic stress is not clear. Since the expression of glutamate transporter can be reduced by chronic stress (Liu et al., 2016, 2017; Zhu et al., 2017), the increase in ambient glutamate after a reduction in transporter function may downregulate exNMDAR expression, which are more sensitive to ambient glutamate than sNMDARs. The loss of astrocytes, a suggested source of glutamate for activating exNMDARs (Carmignoto and Fellin, 2006), in the stressed brain (Czeh et al., 2006) could also contribute to reduced exNMDAR function.

A loss of exNMDAR function could contribute to the impact of chronic stress on hippocampal function. Since exNMDARs mediate NMDAR spikes (Suzuki et al., 2008; Oikonomou et al., 2012) that amplify synaptic signals (Harris and Pettit, 2008) and enhance neuronal excitability (Sah et al., 1989; Oikonomou et al., 2012), reduced exNMDAR function could contribute to the negative impact of chronic stress on rats' performance in spatial working and reference memory tasks (Luine et al., 1994; Henningsen et al., 2009; Veena et al., 2009). However, not all hippocampus-related cognitive functions are impaired by chronic stress. For instance, contextual fear conditioning is enhanced in chronically stressed rats (Conrad et al., 1999; Sandi et al., 2001). Given that exNMDARs are associated with forgetting mechanisms such as long-term depression (Liu et al., 2013), reduction in exNMDARs may be related to the enhanced encoding of aversive information in stressed mice. Recently, we have shown that mice that are susceptible to a chronic

\section{REFERENCES}

Alexander, J. K., DeVries, A. C., Kigerl, K. A., Dahlman, J. M., and Popovich, P. G. (2009). Stress exacerbates neuropathic pain via glucocorticoid and NMDA receptor activation. Brain Behav. Immun. 23, 851-860. doi: 10.1016/j.bbi.2009. 04.001 social defeat stressor show enhanced negative memory engrams formation in the hippocampal CA1 region (Zhang et al., 2019). In addition, we observed a reduction of exNMDAR function in this hippocampal region of mice that are susceptible, but not resilient to this stressor (Tse et al., 2019). These findings suggest that the enhanced encoding of aversive information could be related to a reduction in exNMDARs. Further studies could investigate if enhancing exNMDAR function, for example using drug-coating nanoparticles that are too big to enter synaptic cleft (Savchenko et al., 2016; Tse et al., 2019; Valente et al., 2020), will ameliorate the enhancement of aversive memory in stressed rats.

\section{CONCLUSION}

In conclusion, we show that apart from sNMDAR function, exNMDAR function can be modulated by both acute and chronic stress. Given the emerging functional roles of exNMDARs, changes in exNMDAR function could be related to the pathogenesis of stress-related brain disorders.

\section{DATA AVAILABILITY STATEMENT}

The raw data supporting the conclusions of this article will be made available by the authors, without undue reservation.

\section{ETHICS STATEMENT}

The animal study was reviewed and approved by Facility Animal Care Committee, McGill University.

\section{AUTHOR CONTRIBUTIONS}

YT, MN, and TW conducted all experiments. YT and TW designed the study. YT, AL, and TW analyzed the data and wrote the manuscript. All authors have approved the submitted version.

\section{FUNDING}

This work was supported by the Canadian Institutes of Health Research (137003).

\section{ACKNOWLEDGMENTS}

We would like to thank Alice Wong for her technical assistance.

Brown, E. S., Rush, A. J., and McEwen, B. S. (1999). Hippocampal remodeling and damage by corticosteroids: implications for mood disorders. Neuropsychopharmacology 21, 474-484. doi: 10.1016/s0893-133x(99)00 054-8

Calabrese, F., Guidotti, G., Molteni, R., Racagni, G., Mancini, M., and Riva, M. A. (2012). Stress-induced changes of hippocampal 
NMDA receptors: modulation by duloxetine treatment. PLoS One 7:e37916.

Campbell, S., and Macqueen, G. (2004). The role of the hippocampus in the pathophysiology of major depression. J. Psychiatry Neurosci. 29, 417-426.

Carmignoto, G., and Fellin, T. (2006). Glutamate release from astrocytes as a non-synaptic mechanism for neuronal synchronization in the hippocampus. J. Physiol. Paris 99, 98-102. doi: 10.1016/j.jphysparis.2005.12.008

Christian, K. M., Miracle, A. D., Wellman, C. L., and Nakazawa, K. (2011). Chronic stress-induced hippocampal dendritic retraction requires CA3 NMDA receptors. Neuroscience 174, 26-36. doi: 10.1016/j.neuroscience.2010.11.033

Conrad, C. D., LeDoux, J. E., Magarinos, A. M., and McEwen, B. S. (1999). Repeated restraint stress facilitates fear conditioning independently of causing hippocampal CA3 dendritic atrophy. Behav. Neurosci. 113, 902-913. doi: 10. 1037/0735-7044.113.5.902

Costa-Nunes, J., Zubareva, O., Araujo-Correia, M., Valenca, A., Schroeter, C. A., Pawluski, J. L., et al. (2014). Altered emotionality, hippocampus-dependent performance and expression of NMDA receptor subunit mRNAs in chronically stressed mice. Stress 17, 108-116. doi: 10.3109/10253890.2013.872619

Czeh, B., Simon, M., Schmelting, B., Hiemke, C., and Fuchs, E. (2006). Astroglial plasticity in the hippocampus is affected by chronic psychosocial stress and concomitant fluoxetine treatment. Neuropsychopharmacology 31, 1616-1626. doi: 10.1038/sj.npp.1300982

Duman, R. S., and Monteggia, L. M. (2006). A neurotrophic model for stress-related mood disorders. Biol. Psychiatry 59, 1116-1127. doi: 10.1016/j.biopsych.2006.02.013

Fellin, T., Pascual, O., Gobbo, S., Pozzan, T., Haydon, P. G., and Carmignoto, G. (2004). Neuronal synchrony mediated by astrocytic glutamate through activation of extrasynaptic NMDA receptors. Neuron 43, 729-743. doi: 10.1016/ j.neuron.2004.08.011

Gore, A. C., Yeung, G., Morrison, J. H., and Oung, T. (2000). Neuroendocrine aging in the female rat: the changing relationship of hypothalamic gonadotropin-releasing hormone neurons and N-methyl-D-aspartate receptors. Endocrinology 141, 4757-4767. doi: 10.1210/endo.141.12.7841

Groc, L., Heine, M., Cousins, S. L., Stephenson, F. A., Lounis, B., Cognet, L., et al. (2006). NMDA receptor surface mobility depends on NR2A-2B subunits. Proc. Natl. Acad. Sci. USA 103, 18769-18774. doi: 10.1073/pnas.0605238103

Hammen, C. (2005). Stress and depression. Annu. Rev. Clin. Psychol. 1, 293-319.

Hardingham, G. E., and Bading, H. (2003). The Yin and Yang of NMDA receptor signalling. Trends Neurosci. 26, 81-89. doi: 10.1016/s0166-2236(02)00040-1

Hardingham, G. E., and Bading, H. (2010). Synaptic versus extrasynaptic NMDA receptor signalling: implications for neurodegenerative disorders. Nat. Rev. Neurosci. 11, 682-696. doi: 10.1038/nrn2911

Harris, A. Z., and Pettit, D. L. (2008). Recruiting extrasynaptic NMDA receptors augments synaptic signaling. J. Neurophysiol. 99, 524-533. doi: 10.1152/jn. 01169.2007

Henningsen, K., Andreasen, J. T., Bouzinova, E. V., Jayatissa, M. N., Jensen, M. S., Redrobe, J. P., et al. (2009). Cognitive deficits in the rat chronic mild stress model for depression: relation to anhedonic-like responses. Behav. Brain Res. 198, 136-141. doi: 10.1016/j.bbr.2008.10.039

Karst, H., and Joels, M. (2003). Effect of chronic stress on synaptic currents in rat hippocampal dentate gyrus neurons. J. Neurophysiol. 89, 625-633. doi: 10.1152/jn.00691.2002

Krugers, H. J., Koolhaas, J. M., Bohus, B., and Korf, J. (1993). A single social stressexperience alters glutamate receptor-binding in rat hippocampal CA3 area. Neurosci. Lett. 154, 73-77. doi: 10.1016/0304-3940(93)90174-j

Lau, C. G., and Zukin, R. S. (2007). NMDA receptor trafficking in synaptic plasticity and neuropsychiatric disorders. Nat. Rev. Neurosci. 8, 413-426. doi: 10.1038/ nrn2153

Le Meur, K., Galante, M., Angulo, M. C., and Audinat, E. (2007). Tonic activation of NMDA receptors by ambient glutamate of non-synaptic origin in the rat hippocampus. J. Physiol. 580, 373-383. doi: 10.1113/jphysiol.2006.123570

Li, N., Liu, R. J., Dwyer, J. M., Banasr, M., Lee, B., Son, H., et al. (2011). Glutamate $\mathrm{N}$-methyl-D-aspartate receptor antagonists rapidly reverse behavioral and synaptic deficits caused by chronic stress exposure. Biol. Psychiatry 69, 754-761. doi: 10.1016/j.biopsych.2010.12.015

Li, S., Jin, M., Koeglsperger, T., Shepardson, N. E., Shankar, G. M., and Selkoe, D. J. (2011). Soluble Abeta oligomers inhibit long-term potentiation through a mechanism involving excessive activation of extrasynaptic NR2B-containing
NMDA receptors. J. Neurosci. 31, 6627-6638. doi: 10.1523/jneurosci.0203-11. 2011

Liu, D. D., Yang, Q., and Li, S. T. (2013). Activation of extrasynaptic NMDA receptors induces LTD in rat hippocampal CA1 neurons. Brain Res. Bull. 93, 10-16. doi: 10.1016/j.brainresbull.2012.12.003

Liu, F., Wu, J., Gong, Y., Wang, P., Zhu, L., Tong, L., et al. (2017). Harmine produces antidepressant-like effects via restoration of astrocytic functions. Prog. Neuropsychopharmacol. Biol. Psychiatry 79, 258-267. doi: 10.1016/j.pnpbp. 2017.06.012

Liu, W. X., Wang, J., Xie, Z. M., Xu, N., Zhang, G. F., Jia, M., et al. (2016). Regulation of glutamate transporter 1 via BDNF-TrkB signaling plays a role in the anti-apoptotic and antidepressant effects of ketamine in chronic unpredictable stress model of depression. Psychopharmacology 233, 405-415. doi: 10.1007/s00213-015-4128-2

Lu, W., Man, H., Ju, W., Trimble, W. S., MacDonald, J. F., and Wang, Y. T. (2001). Activation of synaptic NMDA receptors induces membrane insertion of new AMPA receptors and LTP in cultured hippocampal neurons. Neuron 29, 243-254. doi: 10.1016/s0896-6273(01)00194-5

Luine, V., Villegas, M., Martinez, C., and McEwen, B. S. (1994). Repeated stress causes reversible impairments of spatial memory performance. Brain Res. 639, 167-170. doi: 10.1016/0006-8993(94)91778-7

Luscher, C., Malenka, R. C., and Nicoll, R. A. (1998). Monitoring glutamate release during LTP with glial transporter currents. Neuron 21, 435-441. doi: 10.1016/ s0896-6273(00)80552-8

Magarinos, A. M., and McEwen, B. S. (1995). Stress-induced atrophy of apical dendrites of hippocampal CA3c neurons: involvement of glucocorticoid secretion and excitatory amino acid receptors. Neuroscience 69, 89-98. doi: 10.1016/0306-4522(95)00259-1

Martin, K. P., and Wellman, C. L. (2011). NMDA receptor blockade alters stressinduced dendritic remodeling in medial prefrontal cortex. Cereb. Cortex. 21, 2366-2373. doi: 10.1093/cercor/bhr021

McEwen, B. S. (1999). Stress and hippocampal plasticity. Annu. Rev. Neurosci. 22, 105-122. doi: 10.1146/annurev.neuro.22.1.105

McEwen, B. S. (2003). Mood disorders and allostatic load. Biol. Psychiatry 54, 200-207. doi: 10.1016/s0006-3223(03)00177-x

Milnerwood, A. J., Gladding, C. M., Pouladi, M. A., Kaufman, A. M., Hines, R. M., Boyd, J. D., et al. (2010). Early increase in extrasynaptic NMDA receptor signaling and expression contributes to phenotype onset in Huntington's disease mice. Neuron 65, 178-190. doi: 10.1016/j.neuron.2010. 01.008

Mitani, A., and Komiyama, T. (2018). Real-Time processing of two-photon calcium imaging data including lateral motion artifact correction. Front. Neuroinform. 12:98. doi: 10.3389/fninf.2018.00098

Nasca, C., Zelli, D., Bigio, B., Piccinin, S., Scaccianoce, S., Nistico, R., et al. (2015). Stress dynamically regulates behavior and glutamatergic gene expression in hippocampus by opening a window of epigenetic plasticity. Proc. Natl. Acad. Sci. USA 112, 14960-14965. doi: 10.1073/pnas.1516016112

Nie, H., Zhang, H., and Weng, H. R. (2010). Bidirectional neuron-glia interactions triggered by deficiency of glutamate uptake at spinal sensory synapses. J. Neurophysiol. 104, 713-725. doi: 10.1152/jn.00282.2010

Oikonomou, K. D., Short, S. M., Rich, M. T., and Antic, S. D. (2012). Extrasynaptic glutamate receptor activation as cellular bases for dynamic range compression in pyramidal neurons. Front. Physiol. 3:334. doi: 10.3389/fphys.2012. 00334

Pacheco, A., Aguayo, F. I., Aliaga, E., Munoz, M., Garcia-Rojo, G., Olave, F. A., et al. (2017). Chronic stress triggers expression of immediate early genes and differentially affects the expression of AMPA and NMDA subunits in dorsal and ventral hippocampus of rats. Front. Mol. Neurosci. 10:244. doi: 10.3389/fnmol. 2017.00244

Paoletti, P., Bellone, C., and Zhou, Q. (2013). NMDA receptor subunit diversity: impact on receptor properties, synaptic plasticity and disease. Nat. Rev. Neurosci. 14, 383-400. doi: 10.1038/nrn3504

Papouin, T., Ladepeche, L., Ruel, J., Sacchi, S., Labasque, M., Hanini, M., et al. (2012). Synaptic and Extrasynaptic NMDA receptors are gated by different endogenous coagonists. Cell 150, 633-646. doi: 10.1016/j.cell.2012.06.029

Papouin, T., and Oliet, S. H. (2014). Organization, control and function of extrasynaptic NMDA receptors. Philos. Trans. $R$ Soc. Lond. B Biol. Sci. 369:20130601. doi: 10.1098/rstb.2013.0601 
Parsons, M. P., and Raymond, L. A. (2014). Extrasynaptic NMDA receptor involvement in central nervous system disorders. Neuron 82, 279-293. doi: 10.1016/j.neuron.2014.03.030

Petralia, R. S., Wang, Y. X., Hua, F., Yi, Z., Zhou, A., Ge, L., et al. (2010). Organization of NMDA receptors at extrasynaptic locations. Neuroscience 167, 68-87. doi: 10.1016/j.neuroscience.2010.01.022

Sah, P., Hestrin, S., and Nicoll, R. A. (1989). Tonic activation of NMDA receptors by ambient glutamate enhances excitability of neurons. Science $246,815-818$. doi: $10.1126 /$ science. 2573153

Sandi, C., Merino, J. J., Cordero, M. I., Touyarot, K., and Venero, C. (2001). Effects of chronic stress on contextual fear conditioning and the hippocampal expression of the neural cell adhesion molecule, its polysialylation, and L1. Neuroscience 102, 329-339. doi: 10.1016/s0306-4522(00)00484-x

Savchenko, A., Braun, G. B., and Molokanova, E. (2016). Nanostructured Antagonist of Extrasynaptic NMDA Receptors. Nano. Lett. 16, 5495-5502. doi: 10.1021/acs.nanolett.6b01988

Schwendt, M., and Jezova, D. (2000). Gene expression of two glutamate receptor subunits in response to repeated stress exposure in rat hippocampus. Cell Mol. Neurobiol. 20, 319-329.

Shors, T. J., and Mathew, P. R. (1998). NMDA receptor antagonism in the lateral/basolateral but not central nucleus of the amygdala prevents the induction of facilitated learning in response to stress. Learn Mem. 5, 220-230.

Shors, T. J., and Servatius, R. J. (1995). Stress-induced sensitization and facilitated learning require NMDA receptor activation. Neuroreport 6, 677-680. doi: 10.1097/00001756-199503000-00023

Suzuki, T., Kodama, S., Hoshino, C., Izumi, T., and Miyakawa, H. (2008). A plateau potential mediated by the activation of extrasynaptic NMDA receptors in rat hippocampal CA1 pyramidal neurons. Eur. J. Neurosci. 28, 521-534. doi: 10.1111/j.1460-9568.2008.06324.x

Tovar, K. R., and Westbrook, G. L. (1999). The incorporation of NMDA receptors with a distinct subunit composition at nascent hippocampal synapses in vitro. J. Neurosci. 19, 4180-4188. doi: 10.1523/jneurosci.19-10-04180.1999

Tovar, K. R., and Westbrook, G. L. (2002). Mobile NMDA receptors at hippocampal synapses. Neuron 34, 255-264. doi: 10.1016/s0896-6273(02) 00658-x

Tse, Y. C., Bagot, R. C., Hutter, J. A., Wong, A. S., and Wong, T. P. (2011). Modulation of synaptic plasticity by stress hormone associates with plastic alteration of synaptic NMDA receptor in the adult hippocampus. PLoS One 6:e27215. doi: 10.1371/journal.pone.0027215

Tse, Y. C., Lopez, J., Moquin, A., Wong, S. A., Maysinger, D., and Wong, T. P. (2019). The susceptibility to chronic social defeat stress is related to low hippocampal extrasynaptic NMDA receptor function. Neuropsychopharmacology 44, 1310-1318. doi: 10.1038/s41386-019-0325-8

Valente, P., Kiryushko, D., Sacchetti, S., Machado, P., Cobley, C. M., Mangini, V., et al. (2020). Conopeptide-functionalized nanoparticles selectively antagonize extrasynaptic n-methyl-d-aspartate receptors and protect hippocampal neurons from excitotoxicity in vitro. ACS Nano. 14, 6866-6877. doi: 10.1021/acsnano. $0 \mathrm{c} 00866$

Veena, J., Srikumar, B. N., Mahati, K., Bhagya, V., Raju, T. R., and Shankaranarayana Rao, B. S. (2009). Enriched environment restores hippocampal cell proliferation and ameliorates cognitive deficits in chronically stressed rats. J. Neurosci. Res. 87, 831-843. doi: 10.1002/jnr.21907

Wu, Y. W., Grebenyuk, S., McHugh, T. J., Rusakov, D. A., and Semyanov, A. (2012). Backpropagating action potentials enable detection of extrasynaptic glutamate by NMDA receptors. Cell Rep. 1, 495-505. doi: 10.1016/j.celrep.2012. 03.007

Yamamoto, S., Morinobu, S., Fuchikami, M., Kurata, A., Kozuru, T., and Yamawaki, S. (2008). Effects of single prolonged stress and D-cycloserine on contextual fear extinction and hippocampal NMDA receptor expression in a rat model of PTSD. Neuropsychopharmacology 33, 2108-2116. doi: 10.1038/sj.npp.130 1605

Yao, L., Grand, T., Hanson, J. E., Paoletti, P., and Zhou, Q. (2018). Higher ambient synaptic glutamate at inhibitory versus excitatory neurons differentially impacts NMDA receptor activity. Nat. Commun. 9:4000.

Yuen, E. Y., Liu, W., Karatsoreos, I. N., Feng, J., McEwen, B. S., and Yan, Z. (2009). Acute stress enhances glutamatergic transmission in prefrontal cortex and facilitates working memory. Proc. Natl. Acad. Sci. USA 106, 14075-14079.

Yuen, E. Y., Liu, W., Karatsoreos, I. N., Ren, Y., Feng, J., McEwen, B. S., et al. (2011). Mechanisms for acute stress-induced enhancement of glutamatergic transmission and working memory. Mol. Psychiatry 16, 156-170. doi: 10.1038/ mp. 2010.50

Yuen, E. Y., Wei, J., Liu, W., Zhong, P., Li, X., and Yan, Z. (2012). Repeated stress causes cognitive impairment by suppressing glutamate receptor expression and function in prefrontal cortex. Neuron 73, 962-977. doi: 10.1016/j.neuron.2011. 12.033

Zhang, T. R., Larosa, A., Di Raddo, M. E., Wong, V., Wong, A. S., and Wong, T. P. (2019). Negative memory engrams in the hippocampus enhance the susceptibility to chronic social defeat stress. J. Neurosci. 39, 7576-7590. doi: 10.1523/jneurosci.1958-18.2019

Zhu, X., Ye, G., Wang, Z., Luo, J., and Hao, X. (2017). Sub-anesthetic doses of ketamine exert antidepressant-like effects and upregulate the expression of glutamate transporters in the hippocampus of rats. Neurosci. Lett. 639, 132-137. doi: 10.1016/j.neulet.2016.12.070

Conflict of Interest: The authors declare that the research was conducted in the absence of any commercial or financial relationships that could be construed as a potential conflict of interest.

Publisher's Note: All claims expressed in this article are solely those of the authors and do not necessarily represent those of their affiliated organizations, or those of the publisher, the editors and the reviewers. Any product that may be evaluated in this article, or claim that may be made by its manufacturer, is not guaranteed or endorsed by the publisher.

Copyright (c) 2021 Tse, Nath, Larosa and Wong. This is an open-access article distributed under the terms of the Creative Commons Attribution License (CC BY). The use, distribution or reproduction in other forums is permitted, provided the original author(s) and the copyright owner(s) are credited and that the original publication in this journal is cited, in accordance with accepted academic practice. No use, distribution or reproduction is permitted which does not comply with these terms. 